\title{
Entre la disponibilidad y el acceso a la atención médica. La mirada de los enfermos crónicos en condiciones de pobreza
}

\author{
Between the availability and accessibility \\ to health care. The perspective of patients with chronic \\ diseases living in poverty
}

Luz María Tejada-Tayabas¹, Francisco J. Mercado-Martínez²

${ }^{1}$ Licenciada en Enfermería, Doctora en Ciencias de la Salud Pública. Profesora, Facultad de Enfermería, Universidad Autónoma de San Luis Potosí, México. Itejada@uasIp.mx

2 Médico. Doctor en Ciencias Sociales. Profesor, Universidad de Guadalajara, México. fjaviermercado@yahoo.com.mx
RESUMEN La atención a los enfermos crónicos es un problema cada vez más complejo; de allí que la evaluación de los programas dirigidos a este grupo cobre interés creciente. Este trabajo examina la disponibilidad y el acceso a la atención médica desde la perspectiva de quienes viven con padecimientos crónicos y en la pobreza. Se realizó una evaluación cualitativa en una ciudad mexicana. Participaron diez personas con enfermedades crónicas, mayores de 60 años. Se realizaron 45 entrevistas, llevándose a cabo análisis de contenido. Según los hallazgos, tres modelos coexisten en materia de acceso y uso de la atención médica: uno vinculado a la seguridad social; otro a los servicios públicos de salud, y un tercero a los servicios médicos privados. Pero el hallazgo primordial común pone en evidencia la brecha existente entre los recursos sanitarios disponibles y el acceso y uso limitado de la atención médica debido a obstáculos de diversa índole.

PALABRAS CLAVE Enfermedad Crónica; Evaluación de Servicios de Salud; Investigación Cualitativa; Pobreza.

ABSTRACT Health care of people with chronic diseases is a complex issue; hence there is a growing concern to evaluate health programs oriented to such group. This paper examines availability and accessibility to health care from the perspective of those with chronic diseases living in poverty. A qualitative evaluation was carried out in a Mexican city. Ten persons with chronic diseases, and above 60 years, participated in the study. Forty five interviews were carried out; data were content analyzed. According to the findings, there are three models for accessing and using health care: one is linked to the social security; another to public health services, and a third one related to private health services. Nevertheless, the main common finding shows that there is a gap between existing health care resources and the access and a minor utilization of them due to multiple obstacles.

KEY WORDS Chronic IIIness; Health Services Evaluation; Qualitative Research; Poverty. 


\section{INTRODUCCIÓN}

La atención a los enfermos crónicos es un problema complejo, tanto en países de ingresos altos, como medios. La evaluación de los programas dirigidos a esta población es un asunto de interés creciente dado el incremento de tales enfermedades (1), al convertirse en uno de los retos más importantes que enfrentan los sistemas de salud (2), por el aumento en la demanda de atención médica y sus costos cada vez más elevados en un contexto de escasez de recursos (3).

La disponibilidad y el acceso a los servicios de salud han sido temas a los que se ha prestado atención en años recientes en países como México (4). Por ello, los programas y acciones dirigidas a la atención de los enfermos crónicos se han estudiado desde diferentes enfoques con la intención de evaluar el tipo y la calidad de las prestaciones. Entre otros, se destacan las evaluaciones económicas de la atención a las enfermedades crónicas que miden principalmente sus costos directos e indirectos (5), las evaluaciones epidemiológicas que valoran los efectos de los programas de atención o educativos sobre la salud de los enfermos y el control de la enfermedad (6); así como las evaluaciones gerenciales que analizan la satisfacción de los usuarios con la atención (7), valoran el desempeño de los servicios (8), así como su impacto y sus resultados de la atención (9).

No obstante tales avances, poco se ha indagado sobre la perspectiva de los enfermos crónicos en torno al acceso y la disponibilidad de la atención médica. Por ejemplo, pocos trabajos en los países latinoamericanos evalúan los programas o acciones de atención a los enfermos crónicos bajo un enfoque participativo; mientras que algunos de tipo cualitativo estudian la óptica de enfermos crónicos sobre aspectos generales de la atención $(10,11)$, o los resultados del tratamiento prescrito (12). Unos cuantos se centran en la mirada de los profesionales (13) e incluso logran comparar ambas perspectivas (14-16).

El caso mexicano es semejante al de otros países latinoamericanos. Pocos trabajos exploran la perspectiva de la población con enfermedades crónicas, los sujetos enfermos o los usuarios, sobre el acceso y la disponibilidad de los servicios. Los estudios disponibles exami- nan diferentes factores que afectan su accesibilidad, tales como los geográficos, los económicos, los demográficos, los culturales y los asociados con la oferta y calidad de los servicios $(17,18)$.

Por lo anterior, este estudio (a) examina la disponibilidad y el acceso a la atención médica por parte de los enfermos crónicos. En particular nos interesa explorar la perspectiva de aquellos que viven en condición de pobreza.

\section{MATERIAL Y MÉTODOS}

Este trabajo consiste en una evaluación cualitativa, basada en un modelo interpretativo (19), la cual busca comprender y contextualizar las experiencias de los actores involucrados en los programas de salud. El trabajo de campo se realizó desde octubre de 2005 hasta marzo de 2007 en un barrio de San Luis Potosí, México. Esta ciudad es la capital del estado del mismo nombre. El estado cuenta con aproximadamente dos millones y medio de habitantes, de los cuales $55 \%$ viven en la pobreza; la ciudad tiene poco menos de un millón de habitantes y cerca de un 20\% de ellos viven en tal condición (20).

Participaron diez personas con enfermedades crónicas como diabetes, hipertensión, cáncer, epilepsia, cardiopatía y artritis. Tal decisión se tomó por el interés de conocer la cronicidad, más que una enfermedad en particular, dado que quienes viven con padecimientos crónicos comparten rasgos como enfrentar una enfermedad incurable, verse obligados a transformar su forma de vida y requerir tratamientos complejos. Utilizando un muestreo por conveniencia, seleccionamos siete mujeres y tres hombres, mayores de 60 años y con más de 10 años de padecer sus enfermedades. Su edad promedio fue de 69 años. Ninguna tenía un empleo fijo, dos varones estaban pensionados y dos mujeres se dedicaban a las labores domésticas. La mayoría tenían ingresos familiares menores a un salario mínimo (aproximadamente 95 dólares al mes). Se identificaron dos grupos en función de sus ingresos: uno integrado por aquellos con un empleo eventual o pensionados que reciben un ingreso bajo, pero fijo, lo cual les permite satisfacer necesidades básicas como la alimentación y el acceso a los servicios de salud; otro que incluye a 
quienes no cuentan con ingresos fijos debido al desempleo y subempleo por lo cual su dependencia es total.

La información se obtuvo inicialmente mediante entrevistas semiestructuradas. Ante los pobres resultados de las mismas, se procedió a realizar entrevistas abiertas. Esta segunda modalidad fue más exitosa al permitir una mayor libertad para tratar los temas explorados, en tanto las preguntas fueron abiertas y se enfocaron en la enfermedad, lo cual permitió profundizar más en la atención médica. Se hicieron 45 entrevistas, con una duración de 1,30 horas en promedio; 14 fueron grabadas, las restantes fueron registradas como notas de campo. La recolección de la información se concluyó al llegar a la saturación de los datos (21). Al término de las mismas se registraron los temas claves, para luego reconstruir la conversación, tomando como referente a Robles (22). También se realizaron notas metodológicas, descriptivas y analíticas, las cuales se organizaron en temas (23). Las grabaciones fueron transcritas siguiendo reglas específicas de transcripción (24).

Realizamos análisis de contenido estructurado (25), dado que permite la interpretación subjetiva del contenido de los textos mediante pasos sistemáticos de codificación e identificación de temas que posibilitan seguir un método inductivo y llegar a la interpretación en un proceso circular y permanente. La deconstrucción del texto, separación del todo en partes, se hizo desde la lógica del descubrimiento. El análisis se hizo con apoyo del programa Atlas.ti 5.0 (26).

La participación de los sujetos fue voluntaria y bajo consentimiento informado. Se atendieron principios éticos (27), en términos de respetar su autonomía y autodeterminación, garantizar la confidencialidad de la información y lograr cierto beneficio, al ofrecerles atención preferencial en el Centro de Salud con que cuenta la Facultad de Enfermería de la Universidad en el barrio.

\section{RESULTADOS}

\section{EL SISTEMA DE SALUD}

Al igual que en México y en otros países, el sistema de salud en San Luis Potosí se integra por tres tipos de servicios: aquel brindado por instituciones de la seguridad social; otro por la Secretaría de Salud y un tercero por la medicina privada. La seguridad social, que tiene al Instituto Mexicano del Seguro Social (IMSS) como su máximo exponente, brinda atención a cerca del $40 \%$ de la población; éstos son quienes están asegurados, cuentan con empleo, aportan de forma voluntaria o son familiares de un asegurado. La Secretaría de Salud, a su vez, ofrece servicios médicos a casi la mitad de la población; la cual suele no estar asegurada, ni contar con recursos para acudir a la medicina privada. Esta última cubre aproximadamente al $10 \%$ de la población. Desde 2004, con la reforma del sistema sanitario y el establecimiento del Ilamado Seguro Popular de Salud, parte de la población no asegurada obtiene atención médica bajo tal régimen, pero con frecuencia en las mismas instalaciones y con los mismos recursos de la Secretaría de Salud (SS).

Los servicios de salud cercanos al barrio son: cuatro clínicas privadas, varios consultorios particulares y dos farmacias. Una clínica de seguridad social se encuentra ubicada a unos 15 minutos del barrio, sin embargo allí solo se atienden casos de especialidad, por lo que los habitantes del barrio suelen no ser atendidos allí, salvo en casos de urgencia. Existen dos Centros de Salud de la SS, pero localizados en barrios aledaños.

\section{LOS MODELOS DE ACCESO Y UTILIZACIÓN DE LOS SERVICIOS}

El proceso de búsqueda y el acceso a la atención para el manejo de las enfermedades crónicas sigue tres modelos distintos, según se desprende de los discursos de los participantes entrevistados: uno es aquel descrito por quienes tienen acceso a la seguridad social; otro es el de quienes demandan los servicios públicos de salud, y el tercero es el recorrido por aquellos que acuden a los servicios médicos privados. Tales modelos no son inmutables ni excluyentes, los mismos suelen modificarse debido a circunstancias múltiples; entre otras situaciones ocurre cuando la persona enferma pierde su empleo y en consecuencia, el acceso a la seguridad social, por lo cual pasa por lo general a la segunda vía. 


\section{LA BÚSQUEDA Y EL ACCESO A LA ATENCIÓN EN LA SEGURIDAD SOCIAL}

Según los participantes que son derechohabientes del Seguro Social, aun aquellos con un empleo eventual, no deberían enfrentar mayores problemas en la atención médica, debido a que la consulta, los medicamentos, los estudios de diagnóstico y los tratamientos son gratuitos en esta institución. No obstante, todos reportan dificultades y carencias que les impide el acceso a una atención básica, oportuna y de calidad para el manejo de sus padecimientos crónicos. Para ellos, los recursos son insuficientes en el primer nivel de atención, sobre todo por el número limitado de médicos y el incremento de pacientes. Tal situación suele ser ilustrada con los tiempos de espera cada vez más largos, ante todo en la consulta con los especialistas. Jimena, quien padece hipertensión y cardiopatía, comenta tal situación:

...me dan la cita con la cardióloga cada tres meses y con el doctor familiar cada mes, ¿no ve que somos tantos enfermos? Entonces solo así nos toca a todos. $Y$ si voy antes no me atienden, me dicen que vaya hasta que me toque [consulta]. Entonces ni pa' que ir...

Todos reconocen que el Seguro Social debe proveerles los fármacos sin costo; mas son reiterativos al señalar la carencia de medicamentos, lo que les obliga a suspender o posponer el tratamiento al no contar con recursos propios para adquirirlos. Según estos informantes, tal falta de medicamentos les implica trasladarse a la clínica en repetidas ocasiones, con las dificultades consecuentes por el tiempo y los costos del traslado. Marco Antonio señala al respecto:

...en el Seguro [social]..., a veces atienden bien en unas clínicas y en otras no porque falta el medicamento. $Y$ pos ya nomás le dicen a uno "pos no hay" [medicinas] y nada más.

Las personas enfermas con acceso a la seguridad social reconocen que el Seguro dispone de transporte para los pacientes discapacitados y cuenta con ambulancias para el traslado de enfermos. No obstante, tal recurso no está disponible para ellos, lo que les implica trasladarse en el transporte público urbano, con sus dificultades consecuentes al no existir líneas directas a los centros de atención. Si bien los costos son bajos, ello les implica tiempos prolongados, en parte por el traslado, lo que se agrava por los largos tiempos de espera. Rosa María, por ejemplo comenta que acudir a una consulta en el Seguro les puede significar entre siete u ocho horas lo que le implica, además, dejar de trabajar:

\footnotetext{
...hasta allá [la clínica del Seguro Social] hago una hora y media agarrando 2 camiones... Uno tiene que llegar a formar la tarjeta o que alguien me la forme. Llego como a las 11:30 al Seguro y está uno allí hasta bien tarde, lo vienen pasando a uno [a consulta] como a las 4 o 4:30 de la tarde.
}

Las complicaciones son mayores para quienes tienen limitaciones físicas y dependen de otras personas para acudir a los servicios, principalmente por las dificultades para caminar. A éstos les resulta imposible trasladarse a las clínicas usando el transporte público, razón por la cual se ven obligados a ser acompañados por un familiar y utilizar un taxi, en tanto ninguno de los entrevistados cuenta con automóvil. Ante tales circunstancias, algunas personas suelen sustituir la atención médica del Seguro Social por la medicina tradicional y/o acudir a las Farmacias Similares. Esta es una empresa mexicana que, además de consulta médica, ofrece fármacos a bajo costo con principios activos similares a los medicamentos comerciales. Por ejemplo la metformina de $500 \mathrm{mg}$ empleada en el tratamiento de la diabetes se vende a 3,60 dólares estadounidenses, mientras que el fármaco de marca en la misma dosis suele costar 13,20 dólares. El costo del losartan para el tratamiento de la hipertensión cuesta 14.00 dólares en tales farmacias, mientras que el medicamento comercial cuesta el equivalente a 35,50 dólares.

Rosa María, quien solo puede caminar tramos cortos con ayuda de una andadera debido a la artritis, hipertensión e insuficiencia renal, al hacer una evaluación del asunto concluye que le es más económico comprar los medicamentos en tal farmacia que ir al Seguro Social donde se los podrían dar de forma gratuita: 
...cuando se me acaba mi medicina yo les digo a mis hijos "¿pa' que voy yo al Seguro?, ¿pa' estar sentada esperando toda la mañana nomás por la medicina? Mejor acá la compro en [la Farmacia] Similares. [Además] me tienen que llevar en taxi [al Seguro] y... me cobran 120 pesos [aprox. 10 dólares]. ¿Pa' estar sentada todo el santo día? No, me sale más barato comprar la medicina [que ir al Seguro]..."

\section{LA BÚSQUEDA Y EL ACCESO A LOS SERVICIOS PÚBLICOS DE SALUD}

A diferencia del grupo anterior, los enfermos que acuden a los servicios públicos de salud descartan de entrada la asistencia al centro de salud que les corresponde, el cual se encuentra en otro barrio de la ciudad. Y aunque el costo de la consulta sea bajo -aproximadamente 35 pesos (2,50 dólares)- factores como la distancia, los problemas con el transporte público, los prolongados tiempos de espera y la elevada demanda de atención hacen que casi ninguno considere la atención en el centro de salud como una alternativa viable, ya que les implica ocho horas en promedio acceder a una consulta. A ello se refiere Daniel, quien padece hipertensión y glaucoma, por lo cual tiene limitaciones visuales:

...nos levantamos a las cinco de la mañana para irnos hasta el Centro de Salud para alcanzar ficha; si no, ya no me tocó consulta. Es mucho tiempo el que invertimos. El otro día llegué a las seis y ya había gente formada, de pura suerte que me tocó ficha. A las ocho abren el Centro de Salud y dan las fichas y el que no alcanzó, ni modo, tenemos que regresar al día siguiente. Pero eso sí, más temprano...

Estos participantes enfatizan las dificultades económicas para acceder a los servicios del Centro de Salud. Ante la falta de empleo o el subempleo, sus pocos ingresos solo les permiten tener acceso a servicios públicos gratuitos. Sin embargo la situación es particularmente compleja para quienes sufren alteraciones físicas que les afectan la movilidad o padecen enfermedades que demandan enormes recursos, como es el cáncer. En tales casos se suelen combinar múltiples estrategias para obtener los recursos necesarios para la atención. Entre otras, se solicita apoyo económico a organismos gubernamentales, a miembros de la Iglesia y a los familiares. Un caso es el de Senovia, quien padecía cáncer, por lo cual recibía quimioterapia cada tres semanas en un hospital público, los fármacos requeridos tenían un costo promedio de 11.000 pesos (cerca de 900 dólares). En tal caso, el apoyo de organismos gubernamentales, asociaciones civiles, vecinos, familiares y amigos fue fundamental para cubrir los gastos de la hospitalización, los estudios de diagnóstico y el tratamiento. La misma Senovia se refiere a su situación en los siguientes términos:

\section{El Patrimonio [Patronato Pro Paciente} Oncológico] nos ayuda con unos medicamentos y solo con la mitad de los más caros, el DIF [Programa de Desarrollo Integral de la familia] con los más baratos. No nos dan todo, en la quimio [terapia] pasada nos faltaban 600 pesos [cerca de 55 dólares], los vecinos se cooperaron y juntaron 300 pesos y ya pudimos completar...

Además de los gastos de la consulta, los estudios diagnósticos y los tratamientos, las personas enfermas que tienen una discapacidad o padecen condiciones graves se refieren a los gastos del transporte dado que deben trasladarse en taxi, lo cual les genera dificultades económicas severas que afectan incluso su alimentación. Una vez más Senovia ejemplifica cómo los gastos, derivados de la atención a su enfermedad, repercuten en su alimentación y la de su familia, a pesar del apoyo recibido de parte de las asociaciones y sus redes familiares:

...sin almorzar nos vamos [a la consulta]. Cuando llegan centavitos mi hija puede comer.

Sí, porque mi'ja paga en tres lados; paga 'onde me sacan la sangre, paga 'onde me hacen la quimioterapia y luego paga la consulta. Tres veces paga, y yo voy con ella y si le cobran... hay veces que almuerza y hay veces que no. $Y$ así [mismo] en la casa y sin comer; porque, pos oiga ¿con qué?... 


\section{LA BÚSQUEDA Y EL ACCESO A LA MEDICINA PRIVADA}

La búsqueda de atención en donde se combina la seguridad social, los servicios públicos y los servicios privados es una práctica frecuente. Todos los participantes del estudio han acudido a la medicina privada, sobre todo con los médicos generales, (sea en sus consultorios o en las Farmacias Similares). Pero rara vez recurren a los especialistas (cardiología, ortopedia, gastroenterología, oftalmología, etc.) o a los servicios de urgencias u hospitalización en clínicas aledañas a la colonia.

Varias razones son expuestas, por las cuales terminan acudiendo a la medicina privada. Al respecto, no hay diferencias entre quienes tienen acceso a la seguridad social y los usuarios de los servicios públicos de salud. Una de las razones es cuando consideran su condición de urgencia, de vida o muerte, como suelen decir, ante la necesidad de un diagnóstico preciso y la necesidad de resultados inmediatos y efectivos. Otra razón fundamental es porque cuentan con varios hijos que colaboran financieramente para enfrentar la situación. Rosa María, con artritis desde 20 años atrás y derechohabiente del IMSS, explica la forma y razones por las que decidió acudir al médico privado:

...ahora que me he visto mala, se juntaron [mis hijos] y comentaron "no mama, si vas [a consulta] con tu doctor familiar y luego te da el pase con el especialista de menos va a pasar un mes. De aquí a que arreglas el pase y llegas con el especialista y te ve, y luego que estudios y que te programan, pasan de menos 2 o 3 meses". Y [mis hijos] comentaban, "a ver, ¿ustedes creen que así como está orita mi amá va a aguantar tres meses? ¡Pos no!". Y mejor me llevaron con un doctor particular.

Según varios participantes, el acceso a la consulta y la adquisición de medicamentos en las Farmacias Similares ofrece ventajas por su costo reducido y su cercanía. Al respecto suelen valorar positivamente los efectos de la atención privada que reciben al disminuir sus malestares, aunque sea de manera temporal, aunado a la obtención de un diagnóstico inmediato. Otras ventajas son la cercanía de los consultorios y las clínicas, así como los reducidos tiempos de espera, hechos que les facilita el acceso y la posibilidad de tener una atención inmediata. En casi todos los casos destacan la diferencia en cuanto a la rapidez y efectividad del diagnóstico y el tratamiento otorgado por el médico de los servicios privados, respecto al tiempo que implican en el Seguro Social. Camila, quien padece hipertensión y artritis y requiere una nueva operación para extirparle un absceso hepático, cuenta su experiencia al respecto:

\footnotetext{
...hasta mis hijos quieren hacerme mejor los estudios por fuera del Seguro, en lo particular, para que me operen más pronto. Porque [en el Seguro Social] la verdad es más tardado. De aquí a que me dan la consulta y luego que me toca hacerme los estudios y me vuelve a ver el doctor para programarme la operación... No, pos pasa mucho tiempo.
}

En otro caso, quienes son derechohabientes del Seguro Social acuden a la medicina privada para realizarse estudios diagnósticos especializados. Una razón para utilizar tales servicios es para agilizar la atención en el mismo Seguro Social, pues al contar con un diagnóstico, un tratamiento prescrito y los resultados de un estudio, ello les permite regresar al mismo Seguro con esa información y demandar atención inmediata. Jimena, quien padece hipertensión y cardiopatía, comenta su experiencia sobre el particular:

\footnotetext{
...en el Seguro ni me hicieron caso. Decía el doctor que yo no tenía nada... fui al hospital central y ya luego me mandó ese doctor con el cardiólogo. Me hicieron estudios y me dijo que sí, que estaba bien mala. Luego con esos estudios del corazón y con el papel del cardiólogo fui otra vez al Seguro y solo así fue que me hicieron caso y ya el doctor familiar pos entonces vio que... estaba bien mala...
}

No obstante lo anterior, los servicios médicos privados son percibidos en términos negativos en lo referente a lo económico por sus elevados costos, máxime si no se corresponden a una mejoría equivalente o a la obtención de un diagnóstico preciso. Alejandra tiene diabetes e 
hipertensión y desde hace un año padece un dolor causado por la neuropatía diabética. La misma comenta su experiencia con la atención privada a la que acude después de un largo recorrido por los servicios públicos sin recibir, según ella, un diagnóstico preciso y el alivio esperado.

...hemos gastado mucho, como no se imagina, mucho, mucho dinero, en radiografías, en estudios, en análisis y medicina... y yo igual...

\section{DISCUSIÓN}

El propósito de este trabajo ha sido examinar la disponibilidad y el acceso a la atención médica desde la perspectiva de sujetos que viven con padecimientos crónicos y en condiciones de pobreza. Una evaluación de esta naturaleza implica tomar distancia de aquellos análisis que se centran en la óptica de los profesionales o de los expertos en materia de salud. Este acercamiento centrado en la mirada de los sujetos enfermos ha conllevado el uso de estrategias conceptuales y metodológicas capaces de dar cuenta del giro adoptado en torno al tema en cuestión.

El hallazgo primordial de nuestro estudio consiste en mostrar la existencia de vías de acceso distintas a la atención médica entre los enfermos crónicos pobres. Pero también muestra que estos enfermos crónicos viviendo en la pobreza no cuentan con los medios, las condiciones ni los recursos necesarios para acceder y hacer uso de la atención médica disponible. El mismo trabajo, entonces, pone en evidencia la brecha existente entre los recursos materiales y humanos disponibles en materia de salud y el acceso limitado a los mismos debido a incontables y complejos obstáculos según reportan los sujetos participantes. De hecho, los resultados expuestos parecen indicar que los enfermos crónicos que acuden al Seguro Social así como quienes se atienden en los centros de atención pertenecientes a los servicios públicos tienen como destino ineludible la asistencia a la medicina privada.

Hallazgos de esta naturaleza contradicen las declaraciones cotidianas de funcionarios y directivos del sector salud, tanto de México como de otros países, al destacar la existencia de servicios médicos suficientes para atender a la población en lo general, y a los enfermos crónicos en lo particular. Tales declaraciones remiten con frecuencia a resultados de estudios que muestran no solo la existencia de suficientes centros de atención y profesionales calificados para atender las demandas, sino también de altos índices de satisfacción por parte de la población por los servicios recibidos. Empero con frecuencia se alude a los hallazgos de estudios realizados en otros contextos. Entre otros, Douvova (7) y Fernández (8) reportan que la mayoría de la población entrevistada expresa satisfacción con la atención médica recibida; Sosa (28), por su parte, refiere cómo el Seguro Popular en México ha mejorado el acceso de los enfermos diabéticos a la atención y favorecido su control metabólico.

La discrepancia entre los datos reportados por estos autores y los hallazgos aquí presentados pudiera deberse a varios factores. Los estudios aludidos examinan la situación de los usuarios y de quienes son beneficiarios, que suelen tener una visión más favorable que la de quienes han dejado de asistir o no son beneficiarios, como parece ser el caso de los participantes de este estudio. Así mismo el hecho de que este estudio se haya llevado a cabo en las casas de los participantes, mientras que los reportados renglones arriba en las instalaciones médicas, pudo haber influenciado las respuestas de los informantes. Otro factor a tener en mente es que mientras los participantes de algunos estudios previos padecían diversas condiciones mórbidas, en este solo se incluyen quienes padecen enfermedades crónicas, a lo cual habría que agregar su condición de vivir en la pobreza y ser ancianos. De hecho, ser anciano, pobre y con varias enfermedades crónicas puede estar en el centro de la exclusión social en un buen número de países latinoamericanos. En este estudio se evidencia tal exclusión si se considera que los servicios de salud, a pesar de los programas específicos y prioritarios dirigidos a los enfermos crónicos, al parecer no tienen en cuenta las necesidades particulares de este grupo de personas, tales como sus limitaciones físicas o su escasez de recursos.

En palabras de Abadía y Oviedo (29), las experiencias de los enfermos vinculadas a la atención médica, las cuales siguen tres modelos 
distintos según el tipo de servicios a los que pretenden acceder, pueden ser denominadas itinerarios burocráticos. Desde nuestra óptica, optamos por hacer énfasis en distintos modelos de búsqueda y acceso, como los de quienes acuden al Seguro Social, y a los de los servicios públicos de salud que al parecer tienen como destino ineludible acudir a la medicina privada.

Los enfermos de este estudio combinan diferentes tipos de servicios de salud, incluyendo la atención privada aunque les genere gastos. Ello resulta paradójico y aparentemente contrario a lo referido por otros autores. Donabedian (30), autor muy conocido en la región, señala que la calidad de la atención se distribuye de forma diferencial entre los segmentos de la población según su nivel socioeconómico, también que la medicina privada se considera de más calidad en comparación con los otros tipos de servicios, y que los pobres no acceden a ella. Becker (31), por su parte, sostiene que según el nivel socioeconómico de los enfermos crónicos se accede a distintos tipos de servicios y que a mayores ingresos será más factible el acceso a la atención médica privada. Para los participantes de nuestra investigación, y en contra de la opinión de estos expertos, la decisión de utilizar la medicina privada no solo está determinada por las posibilidades financieras de los hijos, sino también por los pobres resultados y satisfacción con los servicios de salud, tanto públicos como de la seguridad social y el reconocimiento de las ventajas que ofrece el sector privado, al cual se acude en ocasiones extraordinarias, específicamente a la consulta general y a los servicios de urgencias, pero siempre con los médicos generales.

El hallazgo sobre la combinación de modelos en la búsqueda y el acceso a los distintos tipos de servicios para obtener una atención médica satisfactoria ha sido reportado en otros estudios. Desde la óptica de los enfermos participantes en este estudio, se asume el compromiso y la responsabilidad de resolver las limitaciones de los servicios de salud, combinando los diferentes tipos de atención y estableciendo estrategias adicionales para lograr el acceso a una atención efectiva para el diagnóstico y el tratamiento de sus enfermedades crónicas.

Para quienes tienen acceso a los servicios de la SS y la seguridad social, les resulta difícil de entender el asunto de la gratuidad de los servicios, sobre todo cuando se tienen dificultades con los recursos básicos como son los medicamentos y el acceso a una consulta médica de calidad, tal como lo ha referido Mercado (32). Tal situación cuestiona las políticas y programas de atención a la salud que manejan discursos de equidad, calidad y democratización de la salud al señalar que los usuarios de los servicios reciben una atención pronta y un trato digno o que no hay familias que se hayan empobrecido por atender su salud (33). La realidad de los enfermos, viejos y pobres, involucrados en este estudio está alejada de tales discursos; más bien, ponen en el centro de atención asuntos como los de la inequidad y la exclusión.

Algunas consideraciones metodológicas merecen destacarse. La realización de entrevistas semiestructuradas, el uso de la grabadora e incluso el que se tomaran notas durante las entrevistas ha sido de poca utilidad en este estudio. Varias pueden ser las razones de ello. Una es la desconfianza de los entrevistados hacia quien entrevistó (b), entre otras razones por ser ajena al barrio y dada su apariencia física que hacía más acentuadas las diferencias. Además de la desconfianza y el temor a represalias por parte de las instituciones de salud, por las dudas sobre el uso que se le pudiera dar a la información, todo pudo haber afectado la calidad de la información recabada. Ello constituye un desafío metodológico ya que implica la necesidad de dedicar más tiempo para obtener la confianza de los informantes, enfatizar el uso de entrevistas abiertas y de un modelo más participativo. A la vez, descartar el uso de la audiograbadora o la realización de notas frente al entrevistado puede favorecer un ambiente más relajado y de reciprocidad, por lo menos durante el tiempo necesario para establecer la confianza y el rapport.

El análisis de contenido estructurado como método flexible, considera como unidad de análisis los textos que se desglosan en segmentos y que expresan sentidos mínimos de contenido en los que se indagan los temas asociados con el objeto de investigación (25). Las notas de campo de las entrevistas no audiograbadas cumplen los criterios como textos de los que puede obtenerse temas para el análisis. El análisis de contenido se realizó con base en una búsqueda 
de códigos dirigidos, mediante un proceso continuo y sistemático de análisis y recolección de datos, lo que permitió contrastar y validar la información disponible tanto en registros de audio como en notas de campo.

La actitud de desconfianza de los entrevistados puede deberse a varias razones. Por una parte, no es habitual que se tome en cuenta la opinión de las personas enfermas, por otra, es evidente la posición subalterna de los enfermos ante las instituciones prestadoras de servicio y los proveedores de atención, lo que los hace percibirse en desventaja frente a quienes les otorgan los servicios de salud y no sentirse en libertad para expresar sus valoraciones. De allí que un estudio interesado en sus puntos de vista pudiera no tener sentido en el contexto en el cual se mueven cotidianamente.

Terminamos señalando que los participantes de este estudio no son representativos de la población enferma del barrio, ni de la ciudad y menos del país o la región. De hecho, nunca se ha tenido tal propósito. Los resultados dan cuenta de la voz de un grupo de personas que comparten la enfermedad, la cronicidad, la vejez y la pobreza; los mismos dan luz sobre la forma como estos sujetos enfrentan la necesidad de acceder a la atención médica. Dada la carencia de estudios en Latinoamérica sobre enfermos crónicos que viven en la pobreza, algunos resultados de este estudio podrían ofrecer pistas para avanzar en la comprensión de millones de personas que viven en condiciones semejantes en la región. Aquí se ha estudiado el tema en un país con un sistema fragmentado de salud; entre otras cosas, será cuestión de examinar en el futuro si los asuntos mencionados son resueltos en forma satisfactoria en aquellos países con sistemas únicos de salud. Pero desde la óptica de los propios enfermos, no de los directivos de los servicios de salud.

\section{AGRADECIMIENTOS}

Nuestro agradecimiento a las personas enfermas que aceptaron participar en el estudio y compartir su visión del mundo. También a los evaluadores de Salud Colectiva por sus comentarios y sugerencias estimulantes. Agradecemos a la Universidad Autónoma de San Luis Potosí (México) por el apoyo financiero parcial recibido del Fondo de Apoyo a la Investigación.

\section{NOTAS FINALES}

a. Una versión anterior de este trabajo fue presentada en el III Congreso Iberoamericano de Investigación Cualitativa en Salud; 6-9 de mayo de 2008; San Juan, Puerto Rico. b. Las entrevistas fueron realizadas por Luz María Tejada-Tayabas, coautora de este artículo.

\section{REFERENCIAS BIBLIOGRÁFICAS}

1. Yach D, Hawkes C, Gould L, Hofman K. The global burden of chronic diseases. Overcoming impediments to prevention and control. JAMA. 2004;291(21):2616-2622.

2. Lòpez-Arellano O, Blanco-Gil J. La polarización de la política de salud en México. Cadernos de Saúde Pública. 2001;17(1):43-54.
3. Barcelo A, Aedo C, Rajphatak S, Robles S. The cost of diabetes in Latin America and the Caribbean. Bulletin of the World Health Organization. 2003;81(1):19-27.

4. Aday LA, Andersen R. Marco teórico para el estudio del acceso a la atención médica. En: White KL. Investigaciones sobre servicios de salud: una antología. Washington: Organización Panamericana de la Salud; 1992. p. 604-613. 
5. Villarreal-Ríos E, Mathew-Quiroz A, GarzaElizondo ME, Núñez-Rocha G, Salinas-Martínez AM, Gallegos-Handal M. Costo de la atención de la hipertensión arterial y su impacto en el presupuesto destinado a la salud en México. Salud Pública de México. 2002;44(1):7-13.

6. Cabrera-Pivaral CE, González-Pérez G, Vega-López MG, Arias-Merino ED. Impacto de la educación participativa en el índice de masa corporal y glicemia en individuos obesos con diabetes tipo 2. Cadernos de Saúde Pública. 2004;20(1):275-281.

7. Doubova SV, Perez-Cuevas R, Zepeda-Arias M, Flores-Hernández S. Satisfaction of patients suffering from type 2 diabetes and/or hypertension with care offered in family medicine clinics in Mexico. Salud Pública de México. 2009;51(3):231-239.

8. Fernández-Cantón S. El IMSS en cifras. Evaluación del desempeño de las instituciones de salud. México, 2004. Revista Médica del Instituto Mexicano del Seguro Social. 2006;44(5):481-488.

9. Montoya JA, Ballesteros AM, Fontcuberta J, García A, Santiago C, Moreno MA. Atención al paciente diabético en un área de salud: ciclo de mejora. Calidad Asistencial. 2003;18(4):203-208.

10. Mercado FJ, Ramos IM, Valdez E. La perspectiva de enfermos crónicos sobre la atención médica en Guadalajara, México. Un estudio cualitativo. Cadernos de Saúde Pública. 2000;16(3):759-772.

11. Infante FA, Proudfoot JG, Powell-Davies G, Bubner TK, Holton $\mathrm{CH}$, Beilby JJ, Harris MF. How people with chronic illnesses view their care in general practice: a qualitative study. Medical Journal Australia. 2004;181(2):70-73.

12. Paskulin LM, Días VR. Como é ser cuidado em casa: as percepções dos clientes. Revista Brasileira de Enfermagem. 2002;55(2):140-145.

13. García MH, Gutiérrez-Cuadra JL, LinerosGonzález C, Ruiz-Barbosa C, Rabadán A. Los pacientes y la calidad de los servicios de atención primaria de salud. Opinión de los profesionales de los centros de salud de la Bahía de Cádiz y la Janda. Atención Primaria. 2002;30(7):425 -434.

14. Mercado FJ, Alcántara E, Lizardi A, Benítez R. Utilización de los servicios de salud en México: perspectivas de los individuos con diabetes. Atención Primaria. 2003;31(1):32-38.

15. Mercado FJ, Alcántara EC, Tejada LM, Lara N, Sánchez A. O atendimento médico aos doentes crônicos no México. Quais as diferenças entre a perspectiva dos médicos, das enfermeiras e das pessoas doentes? En: Magalhães ML, Mercado FJ, editores. Pesquisa qualitativa de serviços de saúde. Petrópolis: Vozes; 2004. p. 363-400.

16. Ruiz A, Mercado FJ, Perea MB. La atención a la enfermedad crónica en los servicios públicos de salud. La perspectiva de los profesionales y los legos. Investigación en Salud. 2006;8(1):22-30.

17. Garza-Elizondo ME, Salinas-Martínez AM, Núñez-Rocha G, Villarreal-Ríos E, MorenoMonsiváis MG. Necesidades de accesibilidad para acciones preventivas. Una perspectiva de la población en Monterrey, México en 2005. Revista Española de Salud Pública. 2008;82(5):547-557.

18. Garza-Elizondo ME, Salinas-Martínez AM, Núñez-Rocha G, Villarreal $E$, Vázquez-Treviño MG, Vásquez-Salazar, MG. Accesibilidad geográfica para detección temprana de enfermedades crónico-degenerativas. Revista Médica de Chile. 2008;136(12):1574-1581.

19. Greene J. Understanding social programs through evaluation. En: Denzin N, Lincoln Y, editores. Handbook of Qualitative Research. Thousand Oaks: Sage; 2000. p. 981-999.

20. Consejo Nacional de Evaluación de la Política de Desarrollo Social. Informe Ejecutivo de Pobreza México 2007. [En línea] México: CONEVAL; 2007. [fecha de acceso 17 de mayo de 2009]. URL disponible en: http://www.coneval.gob.mx/contenido/info_public/1778.pdf

21. Strauss A, Corbin J. Bases de la investigación cualitativa. Técnicas y procedimientos para desarrollar la teoría fundamentada. Medellín: Universidad de Antioquia; 2002.

22. Robles SL. La invisibilidad del cuidado a los enfermos crónicos. Un estudio cualitativo en el barrio de Oblatos. Guadalajara: Universidad de Guadalajara; 2007.

23. Emerson RM, Fretz RI, Shaw LL. Writing ethnographic fieldnotes. Chicago: The University of Chicago Press; 1995.

24. Torres TM. Sangre y azúcar. Representaciones sobre la diabetes de los enfermos crónicos en un barrio de Guadalajara, México: Universidad de Guadalajara; 2004.

25. Hsieh HF, Shannon SE. Three approaches to qualitative content analysis. Qualitative Health Research. 2005;15(9):1277-1288. 
26. Muhr T. ATLAS ti. A prototype for the support of text interpretation. Qualitative Sociology. 1991;14(4):349-371.

27. Murphy E, Dingwall R. Ethics of Ethnography. En: Atkinson P, Coffey A, Delamont S, Lofland J, Lofland L. Handbook of Ethnography. Londres: Sage; 2001. p. 339-351.

28. Sosa-Rubí S, Galárraga O, López-Ridaura R. Diabetes treatment and control: the effect of public health insurance for the poor in México. Bulletin of the World Health Organization. 2009;87(7):512-519.

29. Abadía C, Oviedo D. Bureaucratic Itineraries in Colombia. A theoretical and methodological tool to assess managed-care health care system. Social Science \& Medicine. 2009;68(6):11631170.
30. Donabedian A. La calidad de la atención médica. Definición y métodos de evaluación. México: Prensa Médica Mexicana; 1984.

31. Becker G, Newsom E. Socioeconomic status and dissatisfaction with health care among chronically ill African Americans. American Journal of Public Health. 2003;93(5):742-748.

32. Mercado F. Entre el infierno y la gloria. La experiencia de la enfermedad crónica en un barrio urbano. Guadalajara: Universidad de Guadalajara; 1996.

33. Programa Nacional de Salud 2001-2006. [En línea] México DF: Secretaría de Salud; 2001 [fecha de acceso 17 de mayo de 2009]. URL disponible en: http://www.salud.gob.mx/unidades/evaluacion/publicaciones/pns_2001-2006/pns2001006.pdf

\section{FORMA DE CITAR}

Tejada-Tayabas LM, Mercado-Martínez FJ. Entre la disponibilidad y el acceso a la atención médica. La mirada de los enfermos crónicos en condiciones de pobreza. Salud Colectiva. 2010;6(1):35-45.

Recibido el 30 de noviembre de 2009

Versión final presentada el 6 de enero de 2010

Aprobado el 8 de febrero de 2010 